\title{
MEETING CALENDAR 1989
}

\section{IMAC '89, IMAGE MANAGEMENT AND COMMUNICATION IN PATIENT CARE IMPLEMENTATION AND IMPACT \\ June 4-8, 1989 \\ Washington, D.C.}

For further information, contact: Seong K. Mun, PhD, Department of Radiology, Georgetown University Hospital, 3800 Reservoir Road, Washington, DC 20007.

\section{CAR '89-THIRD INTERNATIONAL SYMPOSIUM \\ ON COMPUTER ASSISTED RADIOLOGY}

June 25-28, 1989

Berlin

For further information, contact: Heinz U. Lemke, $\mathrm{PhD}$, Institute for Technical Computer Science, Technical University, Berlin Sekr CG/FR 3-3, Franklin-strasse 28-29, D-100 Berlin 10. 